\title{
Applied Physics at the Condensed Matter Conference
}

\author{
B.L.H. Wilson, Towcester \\ (Plessey Research (Caswell) Ltd.)
}

There are two major annual EPS conferences in the field of condensed matter. This year the divisional conference has been held at Berlin in March, while the European Solid-State Device Research Conference, ESSDERC, will be held in Aachen in September. The division between basic physics and application is so straightforward that it is easy to forget the need to make the connection between them.

We need to recognise areas where physical phenomena are ripe for exploitation, and to continue basic research on imperfectly understood phenomena that underlie existing or developing technology. Technology also develops new tools for experimentation, and device physics poses new questions on fundamental physics. Sometimes methods developed in one branch of pure physics can be applied in quite different branches of applied physics.

Students need to be made aware of the relevance of their own work to application and the continuing physical interest in applied problems. As most students cannot hope for an academic post it is only too important that they become motivated by industrial work and are not lost to science and engineering. Equally obviously, governments spend large sums on science not just for its purely cultural value but in the hope of practical application. There are many reasons why Europe has been slower than the US and Japan in exploiting its own scientific discoveries - the availability of risk capital, individual expectation and incentives. The one that concerns us here is the efficient flow of information across disciplinary and institutional boundaries. I shall try to illustrate some of these points from papers given at this year's CMD conference in Berlin. If I restrict myself to semiconductors it is not because these applications will necessarily turn out in the end to be the most important, but because they are the most obvious and reflect my own interests.

We are told "it is difficult to make predictions, particularly about the future". I shall avoid the difficulty of identifying this year's growth points for new technology by looking back a few years. P. LeComber (Dundee) reminded us how the presence of hydrogen in the plasma deposition of amorphous silicon ( $\alpha$-Si)

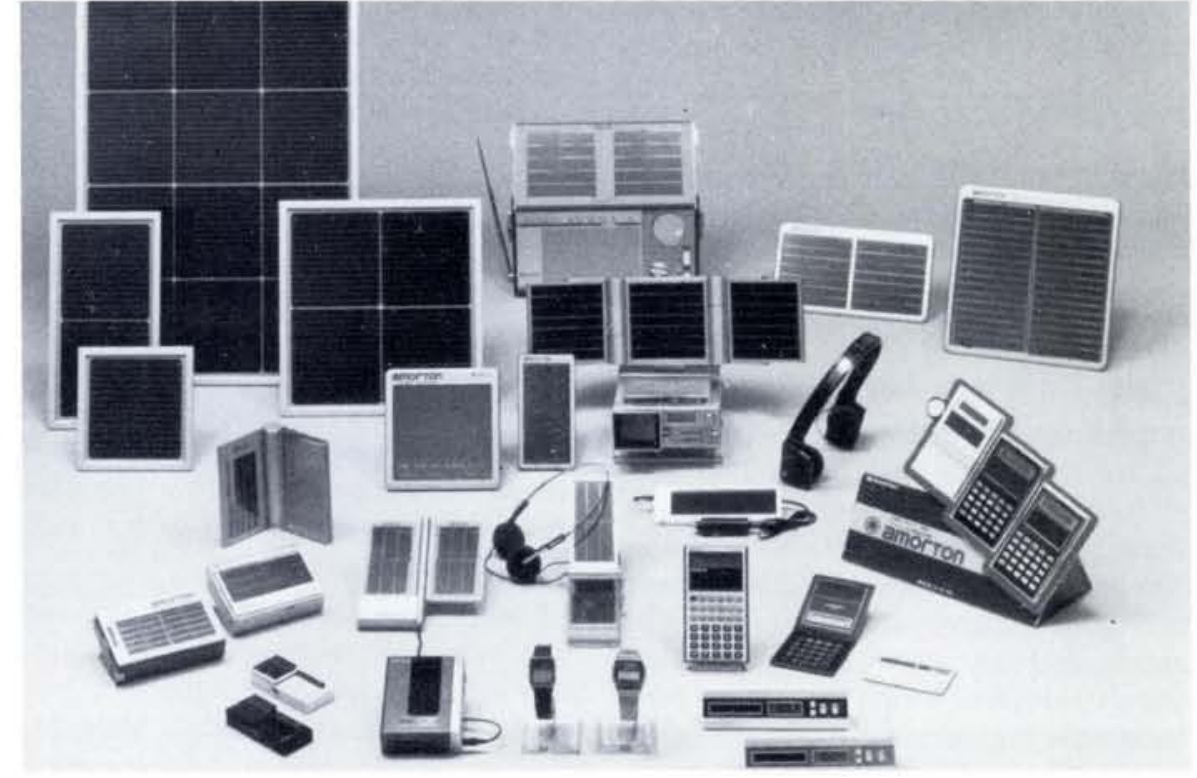

Fig. 1 - An assembly of Japanese products embodying amorphous silicon solar cells in solar panels, calculators, watches and radios. Doping amorphous silicon with hydrogen leads to a reduction in the density of trapping states. Spear and LeComber at Dundee (UK) in 1975 showed that this can lead to $p$-and n-type doped amorphous silicon, and hence $p-n$ junction devices.

(Photo: Sanyo Electric Company) led to fewer deep states associated with dangling bonds, and to the demonstration by Spear and LeComber at Dundee in 1975 of conducting $n$-and p-type $\alpha$-Si by the addition of phosphine and diborane. This achievement was just a part of the growth of understanding of the amorphous state in Europe and the US. (M. Pepper's (Cambridge) Hewlett Packard prize talk showed how this interest continues today but with the focus shifted from 3D to 2D). LeComber went on to speak of some twenty applications of amorphous silicon from elec-

\section{In the Nuclear Physics section of the 17 E 5 Dutch National Institute for Nuclear Phy-

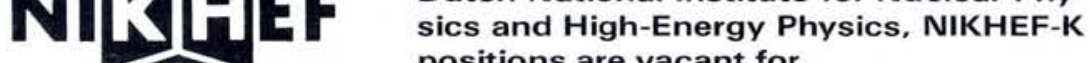 positions are vacant for \\ POST-DOCTORAL RESEARCH ASSOCIATES}

in experimental intermediate energy nuclear physics.

The Institute operates a $500 \mathrm{MeV}$ high-duty cycle linear electron accelerator. The research program focusses on deep-inelast ic particle knock-out processes, high-resolution nuclear-structure studies and the photo-production of pions. An advanced dualspectrometer set-up provides excellent opportunities for coincidence studies with an unmatched energy resolution. The successful candidate will be expected to initiate and conduct research in the electron-scattering team.

The appointment will be for two years.

Further information can be obtained from Prof. Dr. C. de Vries, tel. (20) 5922142.

Applications, including a resume and the names of three referees should be sent within two weeks after appearance of this advertisement to: Prof. Dr. G. van Middelkoop, Scientific Director, NIKHEF-K, P. O. Box 41882, NL-1009 DB Amsterdam. 


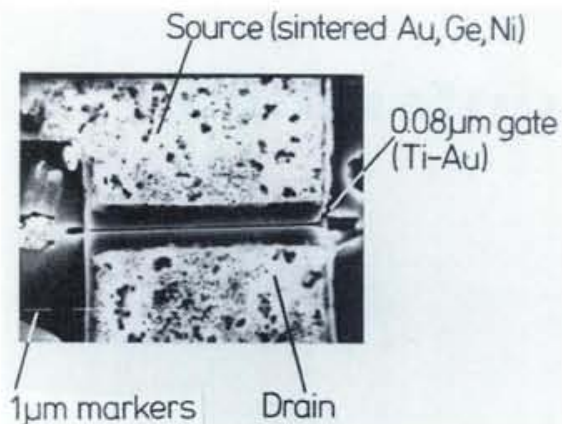

Fig. 2 - An $80 \mathrm{~nm}$ gate length GaAs FET made on special electron beam lithographic equipment at Glasgow University on a wafer with epitaxial layers grown by metallorganic chemical vapour deposition at Plessey Research (Caswell) Ltd.

(Photo: C. Wilkinson, Glasgow University)

trophotography to displays, and how the centre of gravity for application has shifted to Japan. A striking example is the $\alpha$-Si solar cell, where $12 \%$ efficiency has been demonstrated, and in tandem $\alpha$-Si combinations with alloys containing $\mathrm{C}$ and $\mathrm{Ge}, 20 \%$ seems possible within a decade. When the enthusiasm for single crystal solar energy conversion is tempered with economics, such cells remain one of the few real prospects for a serious contribution to world energy supplies from photovoltaic sources.

It is strange that much of the emerging gallium arsenide integrated circuit industry relies on a material, semiinsulating GaAs, which is so poorly understood. While chromium can be used as a deep acceptor, its redistribution during processing can pose problems and the industry is turning to material containing deep stoichiometric donors. Two papers by J.M. Speth (Paderborn) and E. Weber (Berkeley) did not wholly resolve the mystery. While simple antisite defects $A s_{G a}$ seem to exist, DLTS can detect more than one level and optically detected endor spectra show complications, suggesting that more complex structures must be invoked, with $\mathrm{As}_{\mathrm{Ga}}$ as one element. The other antisite defect $\mathrm{Ga}_{\mathrm{As}}$ has not yet been definitely identified but R.C. Newman (Reading) showed how $B_{A s}$ could be identified from its local vibration modes - boron is a frequent but little regarded impurity in $\mathrm{GaAs}$, and is derived from the boric oxide used to cover the melt in crystal pulling.

A similar mystery surrounds the $D(X)$ centres in AIGaAs. R.A. Stradling (London) described how pressure can be used to study donor states in III-V materials. It affects the different conduction sub-bands differently and thus can effect the occupancy of the different donor states associated with each sub-band. The general principles by which pressure or composition affects the appearance of deep states, evidenced by high resistivity or more cogently by absorption or photoluminescence are well understood, although the effects to be expected from different impurities are not. Such effects are vital in the employment of $\mathrm{Al}_{\mathrm{x}} \mathrm{Ga}_{1-\mathrm{x}} \mathrm{As}$ in the high electron mobility transistor, where composition with $x \geq 0.3$, otherwise attractive, may be avoided, because of the deep levels associated with donors. Moreover such deep states appear to undergo relaxation into a metastable state, persistent after the removal of pressure. The speaker did not think these metastable states could be associated with the electronic states of simple donors, but must be associated with lattice relaxation around a complex donor, perhaps associated with hydrogen or oxygen.

The formation of multilayer structures with interfaces, controlled to a monolayer or so, is now commonplace by molecular beam epitaxy (MBE). Mme M. Rezeghi (Orsay) showed how powerful metallorganic chemical vapour deposition (MOCVD) was in this application, particularly for InGaAsP alloys. Many contributed papers described the use of $M B E$ and MOCVD in investigations of luminescence, quantum Hall effect, superlattices and tunnelling in 2D structures, and in injection lasers. D.S. Chemla (Holmdel) showed how a 2D exciton, when first formed possessed virtually no crystal momentum and the narrow exciton line is easily saturated. In times of the order of a picosecond at $300 \mathrm{~K}$ the exciton is scattered to free carrier states, a "reverse relaxation" to higher energies, which subsequently screen the excitonic interaction. The incorporation of the 2D layer in the external cavity of a suitable tuned laser gave rise to a brief easily saturable absorption evidenced by 1.6 ps mode locked laser pulses.

It now seems that we are on the edge of being able to define low dimensional structures by lithography as well as by crystal growth. C. Wilkinson (Glasgow) described his work on nanometer lithography which could give $10 \mathrm{~nm}$ lines on $40 \mathrm{~nm}$ pitch with a $4 \mathrm{~nm}$ realignment accuracy. Although fine structures could be produced on thick substrates, unwanted exposure of resist by back scattered electrons was much reduced by selectively etching a multilayer substrate to a thickness of about $50 \mathrm{~nm}$, and by the use of relatively high energy electrons ( $50 \mathrm{kV}$ ) for exposure. A process of defining lateral quantum wells by locally etching Schottky barriers on an n-type layer was outlined. Some results for $\mathrm{GaAs}$ FETs with gate lengths below $100 \mathrm{~nm}$ were given, with practical implications for FETs above $50 \mathrm{GHz}$, and showing the possibility of better tools for studying hot carrier transport over very short distances.

$A$ wide ranging discussion by $G$. Toulouse (Paris) on Statistical Mechanics of Disorder exemplified the role of the plenary paper in an era of increasing specialisation. There are many practical examples where it is desired to find the absolute minima of a quantity, but the existing solution is stuck at some local minimum. Departures from the local minimum, although initially unfavourable, are needed, and must be weighted by some generalised temperature. Under favourable circumstances, processes similar to annealing can be found to generate lower minima: a familiar example is tapping sugar in a bag to find states of lower potential energy. While Rubik's cube had not been found amenable to such methods, it seemed that it may be useful for problems of component and cell placement, and wiring or routing in electronics, particularly VLSI. H.A. Huberman (Palo Alto) was concerned with the Dynamics of Complexity. Biological systems are notable for their fault tolerance and can adapt, learn and recognise, features which are desired in VLSI. Studies of 2D arrays of simple processors have led to similar self-organising behaviour, learning and self repair.

I hope these few examples will serve to illustrate that technology and application is not just a relaxation for a spare afternoon before we return to 'real' physics, but should be a part even of the most academic physics, posing new problems, offering new solutions, requiring new connections and transcending traditional boudaries.

Fig. 3 - Gallium arsenide ridges $50 \mathrm{~nm}$ high, $30 \mathrm{~nm}$ wide etched by reactive ion etching in freon 12 and oxygen. The marker is 1 micron long. Structures of these dimensions, defined by epitaxy and electron-beam lithography should behave as $1 D$ quantum wells.

(Photo: C. Wilkinson, Glasgow University)

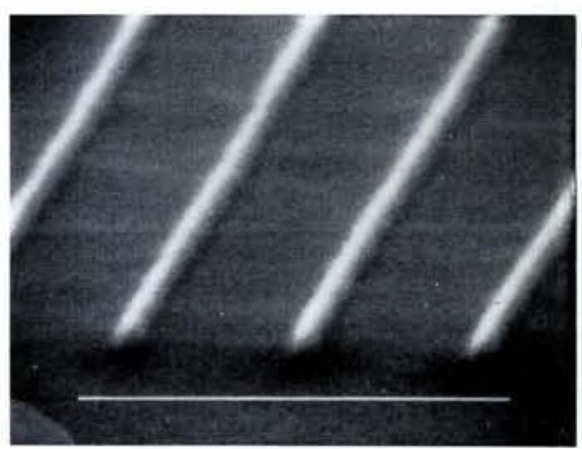

